\title{
Conceptual transfer in preschool children as a function of prior training
}

\author{
ANN M. CLARKE AND G. M. COOPER \\ UNIVERSITY OF HULL, ENGLAND
}

An experiment with normal preschool children indicated that a set to process incoming information cognitively, once established, appeared relatively persistent, if the experimental context was unaltered. Training on an irrelevant complex task had similar transfer effects as training on a relevant task. Subsequent performance in a less structured situation was initially very varied, but again showed the effect of previous training.

Two earlier experiments (Clarke \& Cooper, 1966; Clarke, Cooper, \& Clarke, in press) with young, intellectually normal preschool children showed that training on a complex conceptual sorting task established a cognitive set which greatly enhanced their performance when transferred to an entirely different task. By contrast, training on a very easy task resulted either in slight negative transfer or in results which were no different from those of a control group.

Two questions arose: (1) To what extent would the set induced by training on the complex problem be dissipated if followed by training on an easy problem? (2) To what extent would training on the same conceptual categories as used in the transfer task facilitate its learning if training on the easy problem intervened?

Part 1 of the present paper describes an experiment designed to investigate both issues. Part 2 reports a study concerning the extent to which conceptual categories learned in one situation might enhance performance in another situation demanding entirely different responses.

\section{PART 1}

A group of preschool children were given one trial on the Transfer task. After exclusion of scorers above 11/20, three groups of nine Ss each were closely matched on score, errors, age, sex, and school background, and randomly assigned to treatments. Mean ages and scores were: 50.2 months, 6.6; 51.1 months, 6.4; 50.2 months, 6.5 .

\section{Method}

After allocation to groups, the Ss were trained as follows for two trials per day:

The Relevant group (R) received 24 trials of training on a task similar in construction to the Transfer task, but based on different exemplars, followed immediately by 24 trials on the Easy task, followed in turn by training on the Transfer task.

The Quadrilateral group (Q) received 24 trials on a complex problem based on five quadrilateral shapes, followed by 24 trials on the Easy task, followed by training on the Transfer task.
The Easy group (E) received 24 trials on the Easy task only, prior to training on the Transfer task.

All Ss were trained to a criterion of one score of $20 / 20$ on the Transfer task, with the constraint that all Ss had at least six trials. The apparatus and procedure have been described elsewhere (Clarke \& Cooper, 1966). Five stimulus cards (one from each category) were on permanent display and Ss were required to match the response cards (presented singly in random order) to the appropriate one. The positions of the stimulus cards were varied each trial, and a correction procedure was used. The Transfer task was the same as previously, consisting of five stimulus exemplars and 20 response cards ( 3 x 3 in.), all photographs of common objects within the categories: animals, people, furniture, tableware, and clothes.

The Relevant task consisted of five stimulus exemplars and 15 outline drawings, all of different objects in the same categories as for the Transfer task.

The Quadrilateral task had five stimulus exemplars (a square, rectangle, parallelogram, trapezoid, and irregular shape) and 15 response cards within these categories, each different from the stimulus, because of variations in orientation, size, position on the card, and internal relations of angles and lines.

The Easy task had five stimulus exemplars (a large cross, star, circle, triangle, and crescent) and three identical response cards for each shape, which had merely to be matched on a visual basis.

\section{Results}

On retest, Trial 1 of the Transfer task, the mean scores (out of 20) were as follows: $R-14.67$; $Q-13.33$; $E-10.00 \quad(F=6.15, d f=2 / 24, p<.01$; the comparison $R$ vs $Q$ was nonsignificant, but each differed from $E$ at less than the .01 level). Comparison of $Q$ and $E$ over the first two trials with their respective counterparts in the previous experiment (Clarke, Cooper, \& Clarke, in press) yielded $F$ ratios of less than 1 in each case. It thus appears that the advantage accruing to members of the $Q$ group was not dissipated by training on the Easy task, nor were their scores significantly inferior to those of children who had had Relevant training. The $E$ group had, as before, gained slightly, and no negative transfer was shown.

Trials to reach one score of $20 / 20$ were: $R-3.78$ (range 1-8); Q-4.22 (range 1-9; E-11.44 (range 2-19). A logarithmic transformation was used for the analysis of variance $(F=8.06, d f=2 / 24, p<.005)$. The difference between the $R$ and $Q$ groups was again 
nonsignificant; indeed, of various measures available, the only one which differentiated these was the summation of time to complete six trials, the $R$ group being significantly faster than $Q$ at the $p<.05$ level. Conclusions

The results indicate that: (1) A set to process incoming information cognitively, once established, appeared relatively persistent, granted a similar experimental context. (2) Training on an "irrelevant" complex task (Q) had virtually the same transfer effects as training on the Relevant task, an unexpected finding.

\section{PART 2}

The aim was to discover how far the conceptual schemata formed in the experiment would be available to the children in a much less structured situation. A Control group (C) of 10 children was used in addition to the three experimental groups; one member of the $\mathrm{R}$ and $\mathrm{E}$ groups had by now left the schools. Method and Results

A preliminary warm up procedure was used as follows: Ss were presented with five boxes and a collection containing three different examples of: toy cars, sweets, pens, buttons, elastic bands. Ss were told "Pick up some things which go together and put them in a box, and then pick up some other things which go together and put them in another box, and so on." The number of children per group who sorted correctly immediately was: $\mathrm{R}-4 / 8 ; \mathrm{E}-3 / 8 ; \mathrm{Q}-4 / 9$; $\mathrm{C}-0 / 10$. The majority, therefore, either failed to understand the verbal instructions, or to follow them, but the previously trained Ss nevertheless had an apparent advantage. Most children attempted grouping responses, but failed to use appropriate strategies. All the Ss were shown by demonstration how to do the task, and all then performed it correctly.

Ss were presented with the same five boxes and 15 cards, two photographs (including the previously used stimulus cards), and one outline drawing per category. Thus all the Ss in the three experimental groups had had experience with the photographs, but only the $R$ group had seen the outline drawings (and never in association with the photographs). All Ss were required to name each card, and were instructed as before. Only one child (R group) made a perfect sorting. In each of the four groups there was one or more $S$ who failed altogether to sort on the basis of concepts, either picking up cards randomly or merely separating the outlines from the photographs; eight of the 10 Control Ss scored 0 .

Ss were awarded one point for each picture picked up in conjunction with a related one and placed in the same box (regardless of over- and under-inclusion). Mann-Whitney $U$ tests were performed with these scores and the two-talled $p$ values were: $C$ vs $R, .038$; $C$ vs $Q, .012$, C vs $E, .036$. All others were nonsignificant.

E structured the situation by asking Ss to pick out all the animals, people, clothes, things we use at the table, and furniture (in this order), giving aid as required. Unlike Olver's (1966) finding, that all her three-year-old Ss could readily do this, some Ss had difficulty, either over- or under-including. In all these cases, correct groupings were demonstrated.

Ss were again asked to sort the cards alone. All groups showed an improvement over previous performance, although a few individuals did not. The mean scores were: $\mathrm{C}-5.3 ; \mathrm{E}-9.8 ; \mathrm{Q}-12.2 ; \mathrm{R}-14.0$. The two-tailed $p$ values were: $C$ vs $R-.0018$; $C$ vs $\mathrm{Q}-.012 ; \mathrm{E}$ vs $\mathrm{R}-.064$. All others were nonsignificant. Conclusions

Ability to group the cards conceptually was significantly related to $\mathrm{Ss}^{\prime}$ prior experience with the material. Members of the $\mathrm{R}$ group were most efficient, and Control Ss least so.

Although all members of the three experimental groups had previously achieved $100 \%$ correct performance in a highly structured situation, using the same categories, their initial performance in the less structured situation was extremely varied, suggesting that the strategies required for successful solution in this context were not immediately available to them, but that most could employ superordinate structures once the requirements were understood.

\section{References}

CLARKE, A. M., \& COOPER, G. M. Transfer in category learning of children. Brit. J. Psychol, 1966, 57, 361-373.

CLARKE, A. M., COOPER, G. M., \& CLARKE, A. D. B. Task complexity and transfer in the development of cognitive structures. $J$. exp. Child Psychol, in press.

OLVER, R. R. In J. S. Bruner et al (Ed.), Studies in cognitive growth. New York: Joh Wiley, 1966. P. 82.

Note

1. This research was made possible by a grant from the Nuffield Foundation. 